\title{
A REFINEMENT OF THE KMT INEQUALITY FOR THE UNIFORM EMPIRICAL PROCESS
}

\author{
By David M. Mason ${ }^{1}$ and Willem R. van ZWet \\ University of Munich and University of Leiden
}

\begin{abstract}
A refinement of the Komlós, Major and Tusnády (1975) inequality for the supremum distance between the uniform empirical process and a constructed sequence of Brownian bridges is obtained. This inequality leads to a weighted approximation of the uniform empirical and quantile processes by a sequence of Brownian bridges dual to that recently given by M. Csörgö, S. Csörgő, Horváth and Mason (1986). The present theory approximates the uniform empirical process more closely than the uniform quantile process, whereas the former theory more closely approximates the uniform quantile process.
\end{abstract}

1. Introduction. Let $U_{1}, U_{2}, \ldots$, be a sequence of independent uniform $(0,1)$ random variables, and for each $n \geq 1$, let $G_{n}$ denote the uniform empirical distribution function and $U_{1, n} \leq \cdots \leq U_{n, n}$ the order statistics based on the first $n$ of these uniform $(0,1)$ random variables. Define the uniform empirical quantile function to be, for each $n \geq 1$,

$$
U_{n}(s)=U_{k, n}, \quad(k-1) / n<s \leq k / n, k=1, \ldots, n,
$$

where $U_{n}(0)=U_{1, n}$, and the uniform quantile process

$$
\beta_{n}(s)=n^{1 / 2}\left(s-U_{n}(s)\right), \quad 0 \leq s \leq 1 .
$$

Also let

$$
\alpha_{n}(s)=n^{1 / 2}\left(G_{n}(s)-s\right), \quad 0 \leq s \leq 1
$$

denote the uniform empirical process.

M. Csörgő, S. Csörgő, Horváth and Mason (Cs-Cs-H-M) (1986) recently constructed a probability space on which sit a sequence $U_{1}, U_{2}, \ldots$, of independent uniform $(0,1)$ random variables and a sequence $B_{1}, B_{2}, \ldots$, of Brownian bridges such that for universal positive constants $a, b, c$ and $n_{0}$

$$
P\left(\sup _{0 \leq s \leq d / n} n^{1 / 2}\left|\beta_{n}(s)-B_{n}(s)\right|>a \log d+x\right)<b e^{-c x},
$$

for all $n_{0} \leq d \leq n, 0 \leq x \leq d^{1 / 2}$, with the same inequality holding for the supremum taken over $1-d / n \leq s \leq 1$. Setting $d=n$ in (1) yields the M. Csörgő and Révész (1978) inequality for the Brownian bridge approximation to the uniform quantile process.

\footnotetext{
Received July 1985; revised May 1986.

${ }^{1}$ Research supported by the Alexander von Humboldt Foundation while visiting the University of Munich on leave from the University of Delaware.

AMS 1980 subject classifications. Primary 60F99, 60F17. tion.

Key words and phrases. Weighted empirical and quantile processes, Brownian bridge approxima-
} 
From inequality (1) we obtain immediately that on the Cs-Cs-H-M (1986) probability space

$$
\sup _{0 \leq s \leq 1} n^{1 / 2}\left|\beta_{n}(s)-B_{n}(s)\right|=O(\log n) \quad \text { a.s. }
$$

We note that this particular sequence $B_{n}$ does not approximate the empirical process $\alpha_{n}$ as closely as it does $\beta_{n}$, since by Kiefer (1970)

(3) $\quad \limsup \sup _{n \rightarrow \infty}(2 n)^{1 / 4}\left|\alpha_{n}(s)-\beta_{n}(s)\right| /\left((\log \log n)^{1 / 4}(\log n)^{1 / 2}\right)=1 \quad$ a.s., which in combination with (2) yields

(4) $\quad \limsup \sup _{n \rightarrow \infty}(2 n)^{1 / 4}\left|\alpha_{n}(s)-B_{n}(s)\right| /\left((\log \log n)^{1 / 4}(\log n)^{1 / 2}\right)=1 \quad$ a.s.

Inequality (1) leads to the following important weighted approximation statements [cf. Cs-Cs-H-M (1986)]:

On the Cs-Cs-H-M (1986) probability space we have, for any $0 \leq \nu_{1}<\frac{1}{2}$,

$$
\sup _{1 /(n+1) \leq s \leq n /(n+1)}\left|\beta_{n}(s)-B_{n}(s)\right| /(s(1-s))^{1 / 2-\nu_{1}}=O_{P}\left(n^{-\nu_{1}}\right),
$$

and for any $0 \leq \nu_{2}<\frac{1}{4}$,

$$
\sup _{0 \leq s \leq 1}\left|\alpha_{n}(s)-\bar{B}_{n}(s)\right| /(s(1-s))^{1 / 2-\nu_{2}}=O_{P}\left(n^{-\nu_{2}}\right),
$$

where for $n \geq 2, \bar{B}_{n}(s)=B_{n}(s)$ when $1 / n \leq s \leq 1-1 / n$ and zero elsewhere. It can be shown that statements (5) and (6) do not hold for $\nu_{1} \geq \frac{1}{2}$ and $\nu_{2} \geq \frac{1}{4}$.

The construction of the Cs-Cs-H-M (1986) probability space is based on the Komlós, Major and Tusnády (KMT) (1976) strong approximation to the partial sums of independent random variables. In Cs-Cs-H-M (1986) it was remarked that an analogous theory should be feasible starting out instead from the KMT (1975) strong approximation to the uniform empirical process. The purpose of this paper is to present this alternative theory.

Just as the key result in the Cs-Cs-H-M (1986) theory is inequality (1), a refinement of the M. Csörgö and Révész (1978) inequality, the key result in the present alternative theory is a refinement of the KMT (1975) inequality for the Brownian bridge approximation to the uniform empirical process.

THEOREM 1. There exist a sequence of independent uniform $(0,1)$ random variables $U_{1}, U_{2}, \ldots$, and a sequence of Brownian bridges $B_{1}, B_{2}, \ldots$, sitting on the same probability space $(\Omega, \mathscr{A}, P)$ such that for universal positive constants $C, K$ and $\lambda$,

$$
P\left(\sup _{0 \leq s \leq d / n} n^{1 / 2}\left|\alpha_{n}(s)-B_{n}(s)\right|>C \log d+x\right)<K e^{-\lambda x},
$$

for all $-\infty<x<\infty$ and $1 \leq d \leq n$, with the same inequality holding for the supremum taken over $1-d / n \leq s \leq 1$. 
Setting $d=n$ in (7) yields the original KMT (1975) inequality.

From (7) we have immediately that on the probability space of Theorem 1

$$
\sup _{0 \leq s \leq 1} n^{1 / 2}\left|\alpha_{n}(s)-B_{n}(s)\right|=O(\log n) \quad \text { a.s., }
$$

whereas now by the Kiefer result quoted in (3)

(9) $\quad \limsup \sup _{n \rightarrow \infty}(2 n)^{1 / 4}\left|\beta_{n}(s)-B_{n}(s)\right| /\left((\log \log n)^{1 / 4}(\log n)^{1 / 2}\right)=1$ a.s.

By essentially copying the proofs of Theorems 2.1 and 2.2 of Cs-Cs-H-M (1986), we obtain the following versions of the above weighted approximation statements:

TheOREM 2. On the probability space of Theorem 1, statement (5) holds for all $0 \leq \nu_{1}<\frac{1}{4}$ and statement (6) holds for all $0 \leq \nu_{2}<\frac{1}{2}$.

We see that not only are the almost sure approximation statements reversed, but so are the weighted approximation statements. Hence, we have a theory completely dual to that given in Cs-Cs-H-M (1986). In applications of this approximation methodology in probability and statistics, one now has the choice of working on the Cs-Cs-H-M (1986) probability space or on the probability space of Theorem 1 depending on whether in the particular problem in question one needs to approximate more closely the uniform empirical or the uniform quantile process by a sequence of Brownian bridges. For some of the wide-ranging applications of this weighted approximation theory the reader is referred to Cs-Cs-H-M (1986).

The remainder of this paper is devoted to a proof of Theorem 1. This proof resembles that of the KMT (1975) inequality for the empirical process and it would have been convenient if we could merely have pointed out the modifications needed to produce the refinement of Theorem 1. Unfortunately, the proof in KMT (1975) contains few details and we shall have to provide these in the present paper. The inequality for the tail of a multinomial distribution that is given in Lemma 3, may be of independent interest.

2. Outline of the proof of Theorem 1. Let $B$ denote a fixed Brownian bridge. For each integer $n \geq 1$ we construct $n$ independent uniform $(0,1)$ random variables $U_{1}^{(n)}, \ldots, U_{n}^{(n)}$ as random functions of increments of the Brownian bridge $B$ exactly as in KMT (1975), pages 123-124. Let $\tilde{G}_{n}$ and $\tilde{\alpha}_{n}$ denote the empirical distribution function and empirical process based on $U_{1}^{(n)}, \ldots, U_{n}^{(n)}$. For any nonnegative integers $i$ and $k$ such that $0<(k+1) 2^{-i} \leq 1$, write

$$
\Delta_{i, k}^{(n)}=n\left(\tilde{G}_{n}\left((k+1) 2^{-i}\right)-\tilde{G}_{n}\left(k 2^{-i}\right)\right)
$$

and

$$
D_{i, k}^{(n)}=n^{1 / 2}\left(B\left((k+1) 2^{-i}\right)-B\left(k 2^{-i}\right)\right) .
$$

Also let

$$
\tilde{\Delta}_{i, k}^{(n)}=\Delta_{i+1,2 k}^{(n)}-\Delta_{i+1,2 k+1}^{(n)}
$$


and

$$
\tilde{D}_{i, k}^{(n)}=D_{i+1,2 k}^{(n)}-D_{i+1,2 k+1}^{(n)} .
$$

For the sequence of random vectors $\left(U_{1}^{(n)}, \ldots, U_{n}^{(n)}, B\right), n=1,2, \ldots$, the following fundamental inequality holds:

LEMma 1 [Lemma 2 of KMT (1975)]. There exist positive constants $C_{1}, C_{2}$ and $\eta$ such that

$$
\left|\tilde{\Delta}_{i, k}^{(n)}-\tilde{D}_{i, k}^{(n)}\right| \leq C_{1} 2^{i} n^{-1}\left\{\left(\tilde{\Delta}_{i, k}^{(n)}\right)^{2}+\left(\Delta_{i, k}^{(n)}-n 2^{-i}\right)^{2}\right\}+C_{2},
$$

whenever

$$
\left|\tilde{\Delta}_{i, k}^{(n)}\right| \leq \eta n 2^{-i} \text { and }\left|\Delta_{i, k}^{(n)}-n 2^{-i}\right| \leq \eta n 2^{-i} .
$$

To prove Theorem 1 it will be enough to show that the following inequality is valid:

INEQUALITY 1. There exist universal positive constants $K, C$ and $\lambda$ such that for all $-\infty<x<\infty, n \geq 1$ and $1 \leq d \leq n$,

$$
P\left(\sup _{0 \leq s \leq d / n} n^{1 / 2}\left|B(s)-\tilde{\alpha}_{n}(s)\right|>C \log d+x\right)<K e^{-\lambda x},
$$

with the same inequality holding for the supremum taken over $1-d / n \leq s \leq 1$.

The fact that the second part of Inequality 1 is true follows from the first part and the underlying symmetry of the KMT construction, i.e.,

$$
\left\{\left(\tilde{\alpha}_{n}(s), B(s)\right): 0 \leq s \leq 1\right\}=_{\mathscr{Q}}\left\{\left(\tilde{\alpha}_{n}(1-s), B(1-s)\right): 0 \leq s \leq 1\right\} .
$$

Having established the inequality for $\left(\tilde{\alpha}_{n}, B\right), n=1,2, \ldots$, one can then construct a sequence of independent uniform $(0,1)$ random variables $U_{1}, U_{2}, \ldots$, and a sequence of Brownian bridges $B_{1}, B_{2}, \ldots$, sitting on the same probability space $(\Omega, \mathscr{A}, P)$, say, such that Inequality 1 holds with $\tilde{\alpha}_{n}$ replaced by $\alpha_{n}$ and $B$ by $B_{n}$. The general technique of constructing such a probability space is described in Lemma 3.1.2 in M. Csörgő (1983).

Inequality 1 is almost a direct consequence of the following inequality:

INEQUALITY 2. There exist universal positive constants $a, b$ and $\lambda$ such that for any $n \geq 1$ and $1 \leq z \leq n$,

$$
P\left(\sup _{0 \leq s \leq 2^{-j}} n^{1 / 2}\left|B(s)-\tilde{\alpha}_{n}(s)\right|>z\right)<a \exp \{b(p-j)-\lambda z\},
$$

where $p$ is a nonnegative integer such that

$$
n 2^{-(p+1)}<z / 32 \leq n 2^{-p}
$$

and $j$ is any integer $0 \leq j \leq p$. 
To see that Inequality 2 implies Inequality 1 , we choose any $n \geq 1$ and $1 \leq d \leq n$. Select an $x$ such that $1 \leq C \log d+x \leq n$, where $C=b /(\lambda \log 2)$ with $b$ and $\lambda$ as in Inequality 2 . Define integers $p$ and $j$ by

$$
\begin{gathered}
n 2^{-(p+1)}<(x+C \log d) / 32 \leq n 2^{-p}, \\
j=\min \left(\left[\log _{2}(n / d)\right], p\right),
\end{gathered}
$$

where $[y]$ denotes the integer part of $y$.

Now $d / n \leq 2^{-j}$ and by Inequality 2

$$
\begin{aligned}
& P\left(\sup _{0 \leq s \leq d / n} n^{1 / 2}\left|B(s)-\tilde{\alpha}_{n}(s)\right|>C \log d+x\right) \\
& \quad \leq P\left(\sup _{0 \leq s \leq 2^{-j}} n^{1 / 2}\left|B(s)-\tilde{\alpha}_{n}(s)\right|>C \log d+x\right) \\
& \quad \leq a \exp \{b(p-j)-\lambda C \log d-\lambda x\} .
\end{aligned}
$$

Since $p \leq \log _{2} n+5$, our choice of $C$ and $j$ implies that

$$
b(p-j)-\lambda C \log d \leq b \max \left(\log _{2} d+6,0\right)-b \log _{2} d \leq 6 b
$$

and hence (10) holds for $1 \leq C \log d+x \leq n$ with $K=a \exp \{6 b\}$. If $C \log d+$ $x<1$, then necessarily $x<1$ and (10) holds with $K=\exp \{\lambda\}$.

Finally, let $C \log d+x>n$. There exists a positive $K_{0}$ such that

$$
P\left(\sup _{0 \leq s \leq 1}|B(s)|>r\right)+P\left(\sup _{0 \leq s \leq 1}\left|\tilde{\alpha}_{n}(s)\right|>r\right)<K_{0} \exp \left\{-2 r^{2}\right\},
$$

for all $n \geq 1$ and $r \geq 0$ [cf. M. Csörgő and Révész (1981) and Dvoretzky, Kiefer and Wolfowitz (1956)]. Since now $(C \log d+x)^{2} / n>x$, it follows that (10) holds with $K=K_{0}$ and $\lambda=\frac{1}{2}$. Combining these results we find that Inequality 1 holds, if we assume the validity of Inequality 2.

The proof of Theorem 1 will be complete once we establish Inequality 2 . This will be done in Section 3.

3. Proof of Inequality 2. The proof of Inequality 2 will consist of a number of lemmas. Repeated use will be made of the following special case of Bernstein's inequality: Let $X$ have a binomial distribution with parameters $n \geq 1$ and $0<p<1$. Then for any $r \geq 0$ [cf. Bennett (1962)],

$$
P\left(|X-n p|>(n p)^{1 / 2} r\right)<2 \exp \left\{-\frac{r^{2}}{2\left(1+r /\left(3(n p)^{1 / 2}\right)\right)}\right\} .
$$

For each $i=1,2, \ldots$, set

$$
\xi_{i, n}=\left(n \tilde{G}_{n}\left(2^{-i}\right)-n 2^{-i}\right)^{2}
$$

and for any choice of integers $0 \leq j \leq p$ and $l \geq 1$, define

$$
S_{j, p}=\sum_{i=j+1}^{p+1} 2^{i} \xi_{i, n}, \quad T_{l}=\sum_{i=1}^{l} 2^{2 i-l} \xi_{i, n} .
$$


We shall first be concerned with establishing bounds for the tails of the distributions of $S_{j, p}$ and $T_{l}$.

LeMma 2. For every $A>0$ there exists a positive number $\lambda_{1}$ such that for all $n \geq 1, z \geq 0$ and $p$ so that $z \leq A n 2^{-p}$, and $1 \leq l \leq p+1$,

$$
P\left(n^{-1} T_{l}>z\right)<2 \exp \left\{-\lambda_{1} z\right\} .
$$
tors

Proof. Introduce the independent and identically distributed random vec-

$$
Y_{j}=\left(1_{\left\{U_{j} \leq 2^{-1}\right\}}-2^{-1}, \ldots, 1_{\left\{U_{j} \leq 2^{-l}\right\}}-2^{-l}\right), \quad j=1, \ldots, n,
$$

and the inner product and norm on $\mathbb{R}^{l}$ given by

$$
\langle x, y\rangle=2^{-l} \sum_{i=1}^{l} 2^{2 i} x_{i} y_{i}, \quad\|x\|=\langle x, x\rangle^{1 / 2} .
$$

Notice that

$$
\sigma^{2}=E\left\|Y_{1}\right\|^{2}=\sum_{i=1}^{l} 2^{i-l}\left(1-2^{-i}\right)=2-(l+2) 2^{-l},
$$

so that $\frac{1}{2} \leq \sigma^{2}<2$. By Hölder's inequality we have, for $m \geq 2$,

$$
E\left\|Y_{1}\right\|^{m} \leq \sigma^{2}\left(\sum_{i=1}^{l} 2^{2 i-l}\right)^{(m-2) / 2}<\sigma^{2}\left(2^{(l+1) / 2}\right)^{m-2} .
$$

Applying an exponential bound given by Yurinskiì (1976), page 491, we obtain

$$
\begin{aligned}
P\left(n^{-1} T_{l}>z\right) & =P\left(\left\|\sum_{j=1}^{n} Y_{j}\right\|>(n z)^{1 / 2}\right) \\
& \leq 2 \exp \left\{-\frac{z}{2 \sigma^{2}}\left[1+\frac{1.62}{\sigma^{2}}\left(\frac{2^{l+1} z}{n}\right)^{1 / 2}\right]^{-1}\right\} .
\end{aligned}
$$

Since $2^{l+1} \leq 2^{p+2} \leq 4 A n / z$ and $\frac{1}{2} \leq \sigma^{2} \leq 2$, the lemma follows.

In order to bound the tail of the distribution of $S_{j, p}$ we require a technical lemma which is likely to be of separate interest. Let $\left(X_{1}, \ldots, X_{k+1}\right)$ have a multinomial distribution with parameters $n, p_{1}, \ldots, p_{k+1}$. Assume that $p_{i}>0$ for $i=1, \ldots, k$ and define

$$
s=\sum_{i=1}^{k} p_{i} \in(0,1]
$$

We shall prove

Lemma 3. For every $C>0$ and $\delta>0$, there exist positive numbers $a, b$ and $\lambda$ such that for all $n \geq 1, k \geq 1$ and positive $z, p_{1}, \ldots, p_{k}$ satisfying $z \leq$ 
$C n \min \left\{p_{i}: 1 \leq i \leq k\right\}$ and $s \leq 1-\delta$,

$$
P\left(\sum_{i=1}^{k} \frac{\left(X_{i}-n p_{i}\right)^{2}}{n p_{i}}>z\right)<a \exp \{b k-\lambda z\} .
$$

Proof. For every $\varepsilon>0,(12)$ and the upper bound on $z$ ensure that

$$
\begin{aligned}
P\left(\max _{1 \leq i \leq k} \frac{\left|X_{i}-n p_{i}\right|}{n p_{i}}>\varepsilon\right) & \leq 2 \sum_{i=1}^{k} \exp \left\{-\frac{\varepsilon^{2} n p_{i}}{2(1+\varepsilon / 3)}\right\} \\
& \leq 2 k \exp \{-\lambda(\varepsilon) z\} \leq \exp \{k-\lambda(\varepsilon) z\},
\end{aligned}
$$

with $\lambda(\varepsilon)=\varepsilon^{2}\{2 C(1+\varepsilon / 3)\}^{-1}$. Hence, it suffices to show that

$$
P\left(\sum_{i=1}^{k} Y_{i}>z\right)<a \exp \{b k-\lambda z\}
$$

where

$$
Y_{i}= \begin{cases}\frac{\left(X_{i}-n p_{i}\right)^{2}}{n p_{i}}, & \text { if } \frac{\left(X_{i}-n p_{i}\right)^{2}}{n p_{i}} \leq \varepsilon^{2} n p_{i} \\ 0, & \text { otherwise }\end{cases}
$$

for some constant $\varepsilon>0$ to be chosen below.

Let $\tilde{X}_{1}, \ldots, \tilde{X}_{k+1}$ be independent with $\tilde{X}_{i}$ having a Poisson distribution with parameter $n p_{i}$, and define

$$
\tilde{Y}_{i}= \begin{cases}\frac{\left(\tilde{X}_{i}-n p_{i}\right)^{2}}{n p_{i}}, & \text { if } \frac{\left(\tilde{X}_{i}-n p_{i}\right)^{2}}{n p_{i}} \leq \varepsilon^{2} n p_{i}, \\ 0, & \text { otherwise. }\end{cases}
$$

Clearly there exists $\varepsilon>0$, independent of $n, p_{1}, \ldots, p_{k}$, such that for $i=1, \ldots, k$ and $|h| \leq \frac{1}{2} \varepsilon\left(n p_{i}\right)^{1 / 2}$,

$$
\begin{aligned}
R_{i}(h) & =E \exp \left\{h \frac{\tilde{X}_{i}-n p_{i}}{\left(n p_{i}\right)^{1 / 2}}\right\}=\exp \left\{n p_{i} \exp \left(\frac{h}{\left(n p_{i}\right)^{1 / 2}}\right)-n p_{i}-h\left(n p_{i}\right)^{1 / 2}\right\} \\
& \leq \exp \left\{h^{2}\right\}
\end{aligned}
$$

and this determines our choice of $\varepsilon$. Thus, for $0<y \leq \varepsilon^{2} n p_{i}$ and $h=y^{1 / 2} / 2$,

$$
\begin{aligned}
P\left(\tilde{Y}_{i}>y\right) & \leq P\left(\frac{\tilde{X}_{i}-n p_{i}}{\left(n p_{i}\right)^{1 / 2}}>y^{1 / 2}\right)+P\left(\frac{\tilde{X}_{i}-n p_{i}}{\left(n p_{i}\right)^{1 / 2}}<-y^{1 / 2}\right) \\
& \leq R_{i}(h) \exp \left\{-h y^{1 / 2}\right\}+R_{i}(-h) \exp \left\{-h y^{1 / 2}\right\} \\
& \leq 2 \exp \{-y / 4\} .
\end{aligned}
$$


It follows that

$$
\begin{aligned}
E \exp \left\{\tilde{Y}_{i} / 6\right\} & =\int_{0}^{\infty} P\left(\tilde{Y}_{i}>6 \log x\right) d x \\
& =1+\frac{1}{6} \int_{0}^{\varepsilon^{2} n p_{i}} P\left(\tilde{Y}_{i}>y\right) e^{y / 6} d y \\
& \leq 1+\frac{1}{3} \int_{0}^{\infty} e^{-y / 12} d y=5
\end{aligned}
$$

and hence

$$
\begin{aligned}
P\left(\sum_{i=1}^{k} \tilde{Y}_{i}>z\right) & \leq E \exp \left\{\frac{1}{6}\left(\sum_{i=1}^{k} \tilde{Y}_{j}-z\right)\right\} \\
& \leq \exp \{k \log 5-z / 6\} .
\end{aligned}
$$

The transition from (16) to (15) is achieved by conditioning. We have

$$
\begin{aligned}
P\left(\sum_{i=1}^{k} Y_{i}>z\right) & =P\left(\sum_{i=1}^{k} \tilde{Y}_{i}>z \mid \sum_{i=1}^{k+1} \tilde{X}_{i}=n\right) \\
& =\sum_{m=0}^{n} A_{m} P\left(\sum_{i=1}^{k} \tilde{Y}_{i}>z, \sum_{i=1}^{k} \tilde{X}_{i}=m\right),
\end{aligned}
$$

where

$$
A_{m}=\frac{P\left(\sum_{i=1}^{k} \tilde{X}_{i}=m \mid \sum_{i=1}^{k+1} \tilde{X}_{i}=n\right)}{P\left(\sum_{i=1}^{k} \tilde{X}_{i}=m\right)}=\frac{n !}{(n-m) ! n^{m}}(1-s)^{n-m} e^{n s} .
$$

Application of Stirling's formula in the form

$$
\exp \left\{\frac{1}{12 k+1}\right\} \leq \frac{k ! e^{k}}{(2 \pi k)^{1 / 2} k^{k}} \leq \exp \left\{\frac{1}{12 k}\right\}
$$

to the cases $1 \leq m \leq n-1$ and $m=n$ separately, yields

$$
A_{m} \leq 3\{n /(n-m+1)\}^{1 / 2} \text { for all } 0 \leq m \leq n \text {. }
$$

By considering the ratio $A_{m+1} / A_{m}$ one sees that $A_{m}$ attains its maximum value for $m=[n s]+1$. Hence, for all $0 \leq m \leq n$,

$$
A_{m} \leq 3\left(\frac{n}{n-[n s]}\right)^{1 / 2} \leq 3(1-s)^{-1 / 2} \leq 3 \delta^{-1 / 2} \text {. }
$$

Together with (17) and (16) this implies

$$
P\left(\sum_{i=1}^{k} Y_{i}>z\right) \leq 3 \delta^{-1 / 2} P\left(\sum_{i=1}^{k} \tilde{Y}_{i}>z\right) \leq 3 \delta^{-1 / 2} \exp \{k \log 5-z / 6\} .
$$

The proof is complete. 
LeMma 4. For every $A>0$, there exist positive numbers $a_{2}, b_{2}$ and $\lambda_{2}$ such that for all $n \geq 1, z \geq 0$ and $p$ so that $z \leq A n 2^{-p}$, and $0 \leq j \leq p$,

$$
P\left(n^{-1} S_{j, p}>z\right)<a_{2} \exp \left\{b_{2}(p-j)-\lambda_{2} z\right\} .
$$

Proof. Define

$$
\begin{aligned}
X_{i} & =n\left\{\tilde{G}_{n}\left(2^{-i}\right)-\tilde{G}_{n}\left(2^{-(i+1)}\right)-2^{-(i+1)}\right\}, \quad i=j+1, \ldots, p, \\
X_{p+1} & =n\left\{\tilde{G}_{n}\left(2^{-(p+1)}\right)-2^{-(p+1)}\right\} .
\end{aligned}
$$

For $\nu=j+1, \ldots, p+1$, we have

$$
\begin{aligned}
\xi_{\nu} & =\left(n \tilde{G}_{n}\left(2^{-\nu}\right)-n 2^{-\nu}\right)^{2}=\left(\sum_{i=\nu}^{p+1} X_{i}\right)^{2} \\
& \leq \sum_{i=\nu}^{p+1} 2^{-i / 2} \sum_{i=\nu}^{p+1} 2^{i / 2} X_{i}^{2} \\
& \leq 4\left\{2^{-\nu / 2} \sum_{i=\nu}^{p+1} 2^{i / 2} X_{i}^{2}\right\}
\end{aligned}
$$

and hence

$$
\begin{aligned}
n^{-1} S_{j, p} & \leq 4 n^{-1} \sum_{\nu=j+1}^{p+1} 2^{\nu / 2} \sum_{i=\nu}^{p+1} 2^{i / 2} X_{i}^{2} \\
& \leq 16 n^{-1} \sum_{i=j+1}^{p+1} 2^{i} X_{i}^{2} \\
& \leq 16 n^{-1}\left\{\sum_{i=j+1}^{p} 2^{i+1} X_{i}^{2}+2^{p+1} X_{p+1}^{2}\right\} .
\end{aligned}
$$

Now $\left(X_{j+1}, \ldots, X_{p+1}, n-X_{j+1}-\cdots-X_{p+1}\right)$ has a multinomial distribution with parameters $n, 2^{-(j+2)}, \ldots, 2^{-(p+1)}, 2^{-(p+1)}, 1-2^{-(j+1)}$. Since $z \leq$ $2 A n 2^{-(p+1)}$, application of Lemma 3 for $C=A / 8, k=p-j+1$ and $\delta=\frac{1}{2}$ yields

$$
P\left(n^{-1} S_{j, p}>z\right)<a \exp \{b(p-j+1)-\lambda z / 16\}
$$

and the assertion of the lemma follows.

Choose $\varepsilon>0$. For any integers $p \geq 0,0 \leq l \leq i-1$ and $j \geq 0$, define the events

$$
\begin{aligned}
C_{p}^{(n)} & =\left\{\max _{1 \leq i \leq p+1} 2^{i}\left|\Delta_{i, 0}^{(n)}-n 2^{-i}\right|>\varepsilon n\right\}, \\
C_{i, l}^{(n)} & =\left\{\max _{2^{-l+i} \leq m \leq 2^{l+i+1}-1} 2^{i+1}\left|\Delta_{i+1, m}^{(n)}-n 2^{-(i+1)}\right|>\varepsilon n\right\}, \\
F_{j, p}^{(n)} & =\bigcup_{l=j}^{p-1} \bigcup_{i=l+1}^{p} C_{i, l}^{(n)},
\end{aligned}
$$


where $F_{j, p}^{(n)}=\varnothing$ if $j \geq p$ by convention. Finally, for $0 \leq j \leq p$, set

$$
E_{j, p}^{(n)}=C_{p}^{(n)} \cup F_{j, p}^{(n)} .
$$

Lemma 5. For every $A>0$ and $\varepsilon>0$, there exist positive constants $a_{3}, b_{3}$ and $\lambda_{3}$ such that for all $n \geq 1, z \geq 0$ and $p$ so that $z \leq A n 2^{-p}$, and $0 \leq j \leq p$,

$$
P\left(E_{j, p}^{(n)}\right)<a_{3} \exp \left\{b_{3}(p-j)-\lambda_{3} z\right\} .
$$

Proof. Take $\lambda=\varepsilon^{2} /\{4 A(1+\varepsilon / 3)\}$. As $n 2^{-p} \geq z / A$, inequality (12) yields

$$
P\left(C_{p}^{(n)}\right)<2 \sum_{i=1}^{p+1} \exp \left\{-\lambda z 2^{p-i+1}\right\}<\frac{2 \exp \{-\lambda z\}}{1-\exp \{-\lambda z\}},
$$

which is bounded by $2(1-\exp \{-\lambda\})^{-1} \exp \{-\lambda z\}$ if $z \geq 1$. For $0 \leq z<1$, $P\left(C_{P}^{(n)}\right)<\exp \{\lambda\} \exp \{-\lambda z\}$, so that for $z \geq 0$

$$
P\left(C_{p}^{(n)}\right)<a \exp \{-\lambda z\},
$$

with positive $a$ depending only on $A$ and $\varepsilon$.

For $j=p, F_{j, p}^{(n)}=\varnothing$. For $0 \leq j \leq p-1$, we have

$$
P\left(C_{i, l}^{(n)}\right) \leq 2^{-l+i+1} \exp \{-\lambda z\},
$$

for each $j \leq l \leq p-1$ and $l+1 \leq i \leq p$. Hence, for $0 \leq j \leq p-1$,

$$
P\left(F_{j, p}^{(n)}\right) \leq \sum_{l=j}^{p-1} \sum_{i=l+1}^{p} 2^{-l+i+1} \exp \{-\lambda z\} \leq 2^{p-j+3} \exp \{-\lambda z\},
$$

which completes the proof.

For the proof of our next lemma we need the following combinatorial identity that can be inferred from a similar identity given on page 118 of KMT (1975): Let $f$ be any function on $[0,1]$. For nonnegative integers $i$ and $m$ such that $0<(2 m+1) / 2^{i+1}<1$ define the second differences

$$
\phi(i, m, f)=2 f\left((2 m+1) 2^{-(i+1)}\right)-f\left(m 2^{-i}\right)-f\left((m+1) 2^{-i}\right) .
$$

Then for any choice of nonnegative integers $k, p$ and $l$ such that

$$
2^{-(l+1)}<(2 k+1) 2^{-(p+1)} \leq 2^{-l},
$$

we have

$$
\begin{aligned}
f\left((2 k+1) 2^{-(p+1)}\right)= & \left(2-(2 k+1) 2^{l-p}\right) f\left(2^{-(l+1)}\right) \\
& +\left((2 k+1) 2^{l-p}-1\right) f\left(2^{-l}\right) \\
& +\sum_{i=l+1}^{p} c(i, p, k) \phi(i, k(i), f),
\end{aligned}
$$

where the sum is defined to be zero if $l \geq p$ and for $i=l+1, \ldots, p$,

$$
k(i)=\left[(2 k+1) / 2^{p+1-i}\right], \quad 0 \leq c(i, p, k) \leq 1 .
$$


In addition, we shall use the elementary identity

$$
f\left(2^{-r}\right)=\sum_{i=0}^{r-1} \phi(i, 0, f) 2^{-r+i}, \quad \text { for } r \geq 1,
$$

valid whenever $f(0)=f(1)=0$.

For any two nonnegative integers $j \geq p$, let

$$
\mathscr{I}_{j, p}=\left\{k \geq 0:(2 k+1) 2^{-(p+1)} \leq 2^{-j}\right\} .
$$

Lemma 6. For every $A>0$ there exist positive numbers $a_{4}, b_{4}$ and $\lambda_{4}$ such that for all $n \geq 1, z \geq 0$ and $p$ so that $z \leq A n 2^{-p}$, and $0 \leq j \leq p$,

$$
\begin{aligned}
& P\left(\max _{k \in \mathscr{J}_{, p}} n^{1 / 2}\left|\tilde{\alpha}_{n}\left((2 k+1) 2^{-(p+1)}\right)-B\left((2 k+1) 2^{-(p+1)}\right)\right|>z\right) \\
& \quad<a_{4} \exp \left\{b_{4}(p-j)-\lambda_{4} z\right\} .
\end{aligned}
$$

Proof. Choose positive $C_{1}, C_{2}$ and $\eta$ for which the assertion of Lemma 1 holds and take $\varepsilon=\eta / 2$ in the definition of the event $E_{j, p}^{(n)}$ in Lemma 5 . We shall write $E$ for $E_{j, p}^{(n)}$ and $E^{c}$ for its complement, and we define

$$
Z_{k}=n^{1 / 2}\left|\tilde{\alpha}_{n}\left((2 k+1) 2^{-(p+1)}\right)-B\left((2 k+1) 2^{-(p+1)}\right)\right| .
$$

In view of Lemma 5 it suffices to find positive $a, b$ and $\lambda$ such that

$$
\sum_{k \in \mathscr{J}_{j, p}} P\left(Z_{k}>z, E^{c}\right)<a \exp \{b(p-j)-\lambda z\} .
$$

Obviously, $0 \in \mathscr{I}_{j, p}$; if $k \in \mathscr{I}_{j, p}, k \neq 0$, then $(2 k+1) 2^{-(p+1)}$ cannot be equal to $2^{-l}$ for any $l$. It follows that

$$
\mathscr{I}_{j, p}=\{0\} \cup \bigcup_{l=j}^{p-1} \mathscr{J}_{l}, \quad \mathscr{J}_{l}=\left\{k: 2^{-(l+1)}<(2 k+1) 2^{-(p+1)}<2^{-l}\right\} .
$$

We begin by studying $Z_{0}$. Identity (21) yields

$$
Z_{0} \leq \frac{1}{2} \sum_{i=0}^{p}\left|\tilde{D}_{i, 0}^{(n)}-\tilde{\Delta}_{i, 0}^{(n)}\right| 2^{-(p-i)},
$$

and on the set $E^{c}$ we have

$$
\left|\Delta_{i, 0}^{(n)}-n 2^{-i}\right| \leq \frac{1}{2} \eta n 2^{-i}, \text { for } i=0, \ldots, p+1,
$$

the conclusion for $i=0$ being trivial since $\Delta_{0,0}^{(n)}=n$. This also ensures that on $E^{c}$

$$
\begin{aligned}
\left|\tilde{\Delta}_{i, 0}^{(n)}\right| & =\left|2 \Delta_{i+1,0}^{(n)}-\Delta_{i, 0}^{(n)}\right| \\
& \leq\left|2 \Delta_{i+1,0}^{(n)}-n 2^{-i}\right|+\left|\Delta_{i, 0}^{(n)}-n 2^{-i}\right| \leq \eta n 2^{-i},
\end{aligned}
$$

for $i=0, \ldots, p$ and hence Lemma 1 implies that on $E^{c}$

$$
\begin{aligned}
Z_{0} & \leq C_{1} n^{-1} 2^{-(p+1)} \sum_{i=0}^{p} 2^{2 i}\left\{\left(\tilde{\Delta}_{i, 0}^{(n)}\right)^{2}+\left(\Delta_{i, 0}^{(n)}-n 2^{-i}\right)^{2}\right\}+C_{2} \\
& \leq 5 C_{1} n^{-1} 2^{-(p+1)} \sum_{i=1}^{p+1} 2^{2 i}\left(\Delta_{i, 0}^{(n)}-n 2^{-i}\right)^{2}+C_{2} \\
& =5 C_{1} n^{-1} T_{p+1}+C_{2} .
\end{aligned}
$$


Application of Lemma 2 shows that

$$
P\left(Z_{0}>z, E^{c}\right) \leq 2 \exp \left\{-\lambda_{1}\left(z-C_{2}\right) /\left(5 C_{1}\right)\right\} .
$$

Next we consider the case $k \in \mathscr{J}_{l}$ for some $l$ satisfying $j \leq l \leq p-1$. Since $2^{-(l+1)}<(2 k+1) 2^{-(p+1)}<2^{-l}$, we have $1<(2 k+1) 2^{l-p}<2$ and identities (19) and (21) ensure that

$$
\begin{aligned}
Z_{k} & \leq 2 \sum_{i=0}^{l}\left|\tilde{D}_{i, 0}^{(n)}-\tilde{\Delta}_{i, 0}^{(n)}\right| 2^{-(l-i)}+\sum_{i=l+1}^{p}\left|\tilde{D}_{i, k(i)}^{(n)}-\tilde{\Delta}_{i, k(i)}^{(n)}\right| \\
& \leq 20 C_{1} n^{-1} T_{l+1}+4 C_{2}+\sum_{i=l+1}^{p}\left|\tilde{D}_{i, k(i)}^{(n)}-\tilde{\Delta}_{i, k(i)}^{(n)}\right|
\end{aligned}
$$

where the second inequality follows by the same argument that led to (25). Notice that if $k \in \mathscr{J}_{l}$, then necessarily $2^{p-l}+1 \leq 2 k+1 \leq 2^{p-l+1}-1$ and hence for each $i=l+1, \ldots, p$, we find

$$
2^{-(l+1)} \leq 2 k(i) 2^{-(i+1)}<(2 k(i)+1) 2^{-(i+1)}<2(k(i)+1) 2^{-(i+1)} \leq 2^{-l} .
$$

But this implies that for $i=l+1, \ldots, p$ and on the set $E^{c}$,

$$
\begin{aligned}
\left|\tilde{\Delta}_{i, k(i)}^{(n)}\right| & \leq\left|\Delta_{i+1,2 k(i)}^{(n)}-n 2^{-(i+1)}\right|+\left|\Delta_{i+1,2 k(i)+1}^{(n)}-n 2^{-(i+1)}\right| \\
& \leq \frac{1}{2} \eta n 2^{-i}, \\
\left|\Delta_{i, k(i)}^{(n)}-n 2^{-i}\right| & \leq\left|\Delta_{i+1,2 k(i)}^{(n)}-n 2^{-(i+1)}\right|+\left|\Delta_{i+1,2 k(i)+1}^{(n)}-n 2^{-(i+1)}\right| \\
& \leq \frac{1}{2} \eta n 2^{-i},
\end{aligned}
$$

and Lemma 2 yields

$$
\begin{aligned}
Z_{k} \leq & 20 C_{1} n^{-1} T_{l+1}+4 C_{2} \\
& +C_{1} n^{-1} \sum_{i=l+1}^{p} 2^{i}\left\{\left(\tilde{\Delta}_{i, k(i)}^{(n)}\right)^{2}+\left(\Delta_{i, k(i)}^{(n)}-n 2^{-i}\right)^{2}\right\}+C_{2}(p-l) .
\end{aligned}
$$

Arguing as on page 120 of KMT (1975) it can be shown that

$$
\left\{\left(\Delta_{i, k(i)}^{(n)},\left(\tilde{\Delta}_{i, k(i)}^{(n)}\right)^{2}\right): i=l+1, \ldots, p\right\}=_{\mathscr{D}}\left\{\left(\Delta_{i, 0}^{(n)},\left(\tilde{\Delta}_{i, 0}^{n}\right)^{2}\right): i=l+1, \ldots, p\right\},
$$

and because

$$
\sum_{i=l+1}^{p} 2^{i}\left\{\left(\tilde{\Delta}_{i, 0}^{(n)}\right)^{2}+\left(\Delta_{i, 0}^{(n)}-n 2^{-i}\right)^{2}\right\} \leq 7 \sum_{i=l+1}^{p+1} 2^{i}\left(\Delta_{i, 0}^{(n)}-n 2^{-i}\right)^{2}=7 S_{l, p}
$$

by (24), we obtain for $k \in \mathscr{J}_{l}$,

$$
\begin{aligned}
P\left(Z_{k}>\right. & \left.z, E^{c}\right) \\
\leq & P\left(20 C_{1} n^{-1} T_{l+1}+4 C_{2}>z / 2\right)+P\left(7 C_{1} n^{-1} S_{l, p}+C_{2}(p-l)>z / 2\right) \\
\leq & 2 \exp \left\{-\lambda_{1}\left(z-8 C_{2}\right) /\left(40 C_{1}\right)\right\} \\
& +a_{2} \exp \left\{b_{2}(p-j)-\lambda_{2}\left(z-2 C_{2}(p-l)\right) /\left(14 C_{1}\right)\right\} \\
\leq & \tilde{a} \exp \{\tilde{b}(p-j)-\tilde{\lambda} z\},
\end{aligned}
$$


for positive $\tilde{a}, \tilde{b}$ and $\tilde{\lambda}$. Notice that $\mathscr{J}_{l}$ has $2^{p-l-1}$ elements, so

$$
\sum_{l=j}^{p-1} \sum_{k \in \mathscr{J}_{l}} P\left(Z_{k}>z, E^{c}\right) \leq \tilde{a} \exp \{(\tilde{b}+\log 2)(p-j)-\tilde{\lambda} z\} .
$$

Together with (23) and (26) this yields (22) and the lemma.

For any $0 \leq j \leq p$ and $n \geq 1$, let

$$
\begin{aligned}
& \alpha_{n}(j, p)=\sup _{0 \leq h \leq 2^{-p}} \sup _{h \leq s \leq 2^{-j}} n^{1 / 2}\left|\tilde{\alpha}_{n}(s)-\tilde{\alpha}_{n}(s-h)\right|, \\
& B_{n}(j, p)=\sup _{0 \leq h \leq 2^{-p}} \sup _{h \leq s \leq 2^{-j}} n^{1 / 2}|B(s)-B(s-h)| .
\end{aligned}
$$

LEMMA 7. There exist positive constants $a_{5}, b_{5}$ and $\lambda_{5}$ such that for all $n \geq 1, z>0$ and $p$ so that $n 2^{-(p+1)}<z / 16 \leq n 2^{-p}$, and $0 \leq j \leq p$,

$$
P\left(\alpha_{n}(j, p)+B_{n}(j, p)>z\right)<a_{5} \exp \left\{b_{5}(p-j)-\lambda_{5} z\right\} .
$$

Proof. Whenever $(k-1) 2^{-p} \leq s-h \leq k 2^{-p}$ for some $k=1, \ldots, 2^{p-j}$, then

$$
\begin{aligned}
-n 2^{-p} & \leq n^{1 / 2}\left(\tilde{\alpha}_{n}(s)-\tilde{\alpha}_{n}(s-h)\right) \\
& \leq n^{1 / 2}\left(\tilde{\alpha}_{n}\left((k+1) 2^{-p}\right)-\tilde{\alpha}_{n}\left((k-1) 2^{-p}\right)\right)+2 n 2^{-p} .
\end{aligned}
$$

Because $z / 2>4 n 2^{-p}$ we find by inequality (12)

$$
\begin{aligned}
P\left(\alpha_{n}(j, p)>z / 2\right) & \leq P\left(\alpha_{n}(j, p)>4 n 2^{-p}\right) \\
& \leq 2^{p-j} P\left(n^{1 / 2}\left|\tilde{\alpha}_{n}\left(2^{-(p-1)}\right)\right|>n 2^{-(p-1)}\right) \\
& <2 \exp \left\{(p-j) \log 2-\frac{3 n}{4} 2^{-p}\right\} \\
& \leq 2 \exp \left\{(p-j) \log 2-\frac{3}{64} z\right\} .
\end{aligned}
$$

If $W$ denotes a standard Wiener process, then

$$
\{B(s): 0 \leq s \leq 1\}=_{\mathscr{D}}\{W(s)-s W(1): 0 \leq s \leq 1\},
$$

and hence

$$
\begin{aligned}
P\left(B_{n}(j, p)>z / 2\right) \leq & P\left(\sup _{0 \leq h \leq 2^{-p}} \sup _{h \leq s \leq 2^{-j}} n^{1 / 2}|W(s)-W(s-h)|>z / 4\right) \\
& +P\left(n^{1 / 2} 2^{-p} W(1)>z / 4\right) .
\end{aligned}
$$

It now follows from Lemma 1.2.1 on page 29 of M. Csörgő and Révész (1981) and an elementary bound for the tail of the standard normal distribution [cf. Feller (1968)] that for positive constants $a, b$ and $\lambda$,

$$
P\left(B_{n}(j, p)>z / 2\right)<a \exp \{b(p-j)-\lambda z\} .
$$

Together with (27) this proves the lemma.

We are now in a position to prove Inequality 2 . Choose any $n \geq 1,1 \leq z \leq n$, $p$ so that $n 2^{-(p+1)}<z / 32 \leq n 2^{-p}$ and $0 \leq j \leq p$. Since for every $0 \leq s \leq 2^{-j}$, 
there exists an integer $k \geq 0$ such that $(2 k+1) 2^{-(p+1)} \leq 2^{-j}$ and

we see that

$$
\left|s-(2 k+1) 2^{-(p+1)}\right| \leq 2^{-p},
$$

$$
\begin{aligned}
P\left(\sup _{0 \leq s \leq 2^{-j}} n^{1 / 2}\left|\tilde{\alpha}_{n}(s)-B(s)\right|>z\right) \leq & P\left(\alpha_{n}(j, p)+B_{n}(j, p)>z / 2\right) \\
& +P\left(\max _{k \in \mathscr{S}_{j, p}} n^{1 / 2} \mid \tilde{\alpha}_{n}\left((2 k+1) 2^{-(p+1)}\right)\right. \\
& \left.-B\left((2 k+1) 2^{-(p+1)}\right) \mid>z / 2\right),
\end{aligned}
$$

with $\mathscr{I}_{j, p}$ as in Lemma 6 . As $z / 2$ satisfies the assumptions of Lemma 6 as well as those of Lemma 7, application of these lemmas completes the proof of Inequality 2 and also of Theorem 1.

Acknowledgements. The authors are indebted to Professor F. Götze for a very helpful discussion and to Professor S. Csörgö for pointing out an oversight in our original statement of (3). They thank Professor Gaenssler for his interest and the Associate Editor and the referee for some useful comments.

\section{REFERENCES}

Bennett, G. (1962). Probability inequalities for the sum of independent random variables. J. Amer. Statist. Assoc. 57 33-45.

Csörgö, M. (1983). Quantile Processes with Statistical Applications. SIAM, Philadelphia.

Csörgö, M., Csörgö, S., HorvÁth, L. and Mason, D. M. (1986). Weighted empirical and quantile processes. Ann. Probab. 14 31-85.

Csörgö, M. and RÉvÉsz, P. (1978). Strong approximations of the quantile process. Ann. Statist. 6 882-894.

Csörgő, M. and Révész, P. (1981). Strong Approximations in Probability and Statistics. Academic, New York-Akadémai Kiadó, Budapest.

Dvoretzky, A., Kiefer, J. and Wolfowitz, J. (1956). Asymptotic minimax character of the sample distribution function and of the multinomial estimator. Ann. Math. Statist. 27 642-669.

Feller, W. (1968). An Introduction to Probability Theory and Its Applications 1, 3rd ed. Wiley, New York.

Kiefer, J. (1970). Deviations between the sample quantile process and the sample df. In Nonparametric Techniques in Statistical Inference (M. L. Puri, ed.) 299-319. Cambridge Univ. Press, Cambridge.

Komlós, J., MAJOR, P. and Tusnády, G. (1975). An approximation of partial sums of independent rv's and the sample df. I. Z. Wahrsch. verw. Gebiete 32 111-131.

Komlós, J., MAJOR, P. and TUSNÁDY, G. (1976). An approximation of partial sums of independent rv's and the sample df. II. Z. Wahrsch. verw. Gebiete 34 33-58.

YURINskǐ̆, V. (1976). Exponential inequalities for sums of random vectors. J. Multivariate Anal. 6 473-499.

Department of Mathematical

SCIENCES

501 Ewing Hall

UNIVERSITY OF DELAWARE

Newark, Delaware 19716
UNIVERSITY OF LEIDEN

DEPARTMENT OF MATHEMATICS AND COMPUTER SCIENCE

P.O. Box 9512

2300 RA LEIDEN

The NetherLaNDS 